\title{
Ferramentas Tecnológicas e Metodologias de Apoio à Aprendizagem Personalizada no Ensino Superior: uma Revisão Sistemática
}

\section{Technological Tools and Supporting Methodologies for Personalized Learning in Higher Education: a Systematic Review}

\author{
José Luiz Machado Morais \\ Instituto de Ciência e Tecnologia - Universidade Federal de São Paulo \\ Arlindo Flavio da Conceição \\ Instituto de Ciência e Tecnologia - Universidade Federal de São Paulo
}

\begin{abstract}
Resumo: Tecnologias educacionais e e-learning são facilitadores da personalização do processo de aprendizagem e da promoção do self-paced learning. O objetivo deste estudo é analisar os desafios e os pontos positivos das implementações e modelos que abordam esses conceitos e propor funcionalidades para uma ferramenta de apoio à aprendizagem. Para isso, foi feita uma revisão sistemática da literatura seguindo a metodologia de Kitchenham. Foram selecionados 31 artigos de 4 bases internacionais, os quais foram classificados segundo conceitos, metodologias, plataformas, pontos positivos e desafios apresentados. A análise indicou o blended learning como metodologia mais abordada, a flexibilidade como ponto positivo mais citado e a falta de interação como maior desafio encontrado. Os resultados mostraram que há elementos de e-learning presentes em universidades do mundo todo e que ferramentas tecnológicas de apoio são eficientes na personalização e melhoria do processo de aprendizagem.
\end{abstract}

Palavras-chave: Ensino Superior. Self-paced learning. E-learning.

\begin{abstract}
Educational technologies and e-learning facilitate the customization of the learning process and help to promote self-paced learning. This study aims to analyze the benefits and challenges of models and implementations that use these concepts and also to propose features for a learning supporting tool. A systematic literature review was made following the Kitchenham's methodology, selecting 31 articles from 4 international digital libraries, which were classified by concepts, methodologies, platforms, benefits and challenges. The analysis names blended learning as the most used methodology, flexibility as the most cited advantage and leak of interaction as the biggest challenge. The results show that there are e-learning elements in universities all around the globe and that technological supporting tools are efficient to customize and improve the learning process.
\end{abstract}

Keywords: Higher Education. Self-paced learning. E-learning. 
MURAIS Jose Luiz Machado; DA CONCEICAU, A. F.. Ferramentas Tecnologicas e Metodologias de Apoio a Aprendizagem Personalizada no Ensino Superior: uma Revisáo Sistemática. Informática na Educação: teoria \& prática, Porto Alegre, v.
21, n. 3, p. 124-140, set./dez. 2018. 


\section{Introdução}

Com o advento da tecnologia e a crescente abrangência da Internet, surgem cada vez mais meios e ferramentas para personalizar o processo de aquisição e disseminação do conhecimento. Os conceitos de e-learning e self-paced learning estão presentes em todos os níveis de aprendizagem (CLARK e MAYER, 2003), inclusive no Ensino Superior. Assim, é necessário estudar e analisar os conceitos e metodologias para compreender o funcionamento dessas ferramentas e avaliar se seus objetivos são alcançados, uma vez que tornar a educação de qualidade acessível para o maior número possível de pessoas é um dos desafios enfrentados no século XXI. Um exemplo concreto que já é realidade há cerca de cinco anos, no contexto do Ensino Superior, são os cursos online oferecidos pelo Instituto de Tecnologia de Massachusetts (MIT, do inglês Massachusetts Institute of Technology). O resultado da iniciativa de uma das instituições mais respeitadas do mundo, ao oferecer cursos gratuitos online e levar a excelência do seu ensino a um nível global, mostra a importância da democratização do conhecimento e como a tecnologia é fundamental para esse processo. Pessoas do mundo todo, desde aquelas com mais conhecimento adquirido até as que ainda estão iniciando seus estudos, têm acesso aos materiais fornecidos e podem fazer parte desse processo de aprendizagem que, segundo estudos do próprio MIT (CHANDLER, 2014), é eficiente para todos, independentemente do grau prévio de instrução.

Considerando a implementação de metodologias que abordam o conceito de e-learning, no ano de 2012 surgiram os MOOCs - cursos massivos e abertos online, do inglês Massive Open Online Courses. Dentre as plataformas existentes, as mais conhecidas são o edX (https:// www.edx.org/) e o Coursera (https://www.coursera.org/). A primeira, como mencionado, é uma plataforma online criada em 2012 pelo MIT em parceria com a Universidade de Harvard para promover a aprendizagem online e para oferecer cursos de alta qualidade, das melhores universidades para alunos espalhados por todo o mundo (edX Inc., 2018). Já a segunda, que também foi fundada em 2012 por Daphne Koller e Andrew Ng, dois professores de ciência da computação da Universidade de Stanford, promove um ambiente de aprendizagem no qual o aluno conta com recursos de vídeo, atividades de avaliação por pares, autoavaliação e fóruns de discussão (Coursera Inc., 2018). A ideia é democratizar o acesso ao conhecimento e torná-lo disponível a qualquer hora, em qualquer lugar do mundo.

A educação é fundamental para o desenvolvimento da sociedade. Portanto, é preciso estudar o processo de ensino e aprendizagem e repensar como ele se dará no futuro, sobretudo, na presença de novas tecnologias de comunicação e informação, que são hoje essenciais para produção e difusão eficientes de conteúdos educativos. (MITRA, 2016).

Portanto, dados esses desafios, o objetivo deste artigo é realizar uma revisão sistemática da literatura sobre os métodos de e-learning que promovem o conceito de self-paced learning no contexto do Ensino Superior. A escolha do Ensino Superior como contexto do estudo se deu principalmente por dois fatores: sua importância como base para a vida adulta do indivíduo, seja ela profissional ou acadêmica, que influencia diretamente na sociedade, inclusive na formação de outras pessoas, e o advento dos MOOCs. Dadas as metodologias e ferramentas utilizadas, é importante saber quais delas são eficientes e em que situações podem ser aplicadas. Como o surgimento dos MOOCs se deu em 2012 e eles representaram o surgimento de uma nova forma de aplicar o conceito de e-learning e de promover o self-paced learning, foram considerados apenas artigos publicados a partir desse ano. Dessa forma, este trabalho traz conceitos importantes na área estudada, considerando as metodologias e ferramentas aplicadas, a forma como são implementadas e, quando possível, a eficiência obtida a partir do seu uso. Espera-se, com essa revisão, trazer contribuições significativas para a análise da evolução do processo de aprendizagem na educação e, futuramente, desenvolver uma ferramenta de apoio à aprendizagem no contexto do Ensino Superior que promova o self-paced learning, considerando os principais desafios e pontos positivos e negativos levantados.

Este artigo está organizado da seguinte forma: a Seção 2 apresenta os conceitos fundamentais sobre o estudo; a Seção 3 descreve a metodologia de revisão sistemática 
utilizada no estudo; a Seção 4 mostra os resultados obtidos a partir da análise dos artigos selecionados; a Seção 5 discute os desdobramentos dos resultados encontrados; e, por fim, a Seção 6 traz as considerações finais e a visão de trabalhos futuros.

\section{Conceitos Fundamentais}

O principal conceito no qual se fundamenta esta revisão sistemática da literatura é o selfpaced learning. Este termo descreve a realização do processo de aprendizagem no ritmo de cada aluno (MOTA e SCOTT, 2014; MOTA, 2014). Com a utilização da tecnologia, principalmente dos dispositivos móveis com acesso à Internet, é possível que cada indivíduo aprenda quando e onde quiser, em seu próprio ritmo e do seu próprio jeito, priorizando e enfatizando as suas áreas de maior interesse ou dificuldade. Assim, todas as demais técnicas, metodologias e conceitos abordados neste artigo são vistos como meios digitais e tecnológicos de promover 0 self-paced learning, pois a ideia é priorizar o aluno e explorar ao máximo o seu potencial de aprendizagem.

Quanto às tecnologias de apoio ao ensino e apredizagem, estas estão essencialmente atreladas a alguns conceitos que sempre são citados quando o assunto é a educação. $O$ mais básico deles, que está diretamente relacionado aos demais, é o e-learning. Este termo pode ser definido como um processo de aprendizagem com base na tecnologia que consiste na entrega de conteúdo para os alunos de forma remota por meio de uma rede de computadores (ZHANG et al., 2004). Há ainda os conceitos de e-learning síncrono e assíncrono (HRASTINSKI, 2008). No primeiro, os alunos e o professor ou instrutor interagem em tempo real, por meio de videoconferências e chats online, por exemplo. Já no segundo, não há a necessidade de que todas as partes estejam envolvidas simultaneamente nas atividades, como na troca de mensagens em um fórum ou por e-mail.

A partir do e-learning, é possível chegar a métodos concretos que se apresentam como uma alternativa ao aprendizado clássico em sala de aula e que facilitam a promoção do self-paced learning, como é o caso do blended learning. Este termo refere-se a uma metodologia de aprendizagem na qual são inseridos elementos de e-learning no ensino tradicional (OSORIO GÓMEZ e DUART, 2012) com o objetivo de facilitar o aprendizado do aluno, aumentando o leque de recursos e ferramentas disponíveis, além de estender o processo para fora das salas de aula e, por consequência, promover a personalização do ambiente de aprendizagem. Um exemplo claro de blended learning é a ideia de inverter o ensino do conteúdo em sala de aula e as tarefas de casa, que caracteriza o modelo de flipped learning (BERGMANN e SAMS, 2012). Nesse método, o aluno se dedica a aprender a teoria em casa, antes da aula, por meio de materiais entregues pelo professor, como vídeos, artigos e livros. Feita esta preparação, o aluno aproveita o tempo em sala de aula, com o auxílio do professor, para realizar exercícios e atividades práticas, o que normalmente seria feito em casa. Esse método tem como princípio a ideia de que o aluno necessita mais da presença física do professor durante a aplicação do conhecimento do que na fase da aprendizagem teórica, quando ele pode trabalhar em seu próprio ritmo para assimilar o conhecimento.

Na mesma linha de pensamento do blended learning, existe o hybrid learning, que é um termo que se refere à integração entre e-learning e o ensino tradicional dada por meio do cruzamento de elementos desses dois modelos, que se tornam completamente integrados e inseparáveis, inviabilizando a distinção de suas partes componentes (OSORIO GOMÉZ e DUART, 2012). Este método difere-se do blended learning porque, no segundo, é possível identificar claramente quais são os elementos combinados para a efetivação do processo de aprendizagem, mas ambos podem ser utilizados para a promoção do self-paced learning.

Outra forma que caracteriza o uso do e-learning é o gamification, que pode ser definido como o uso de elementos presentes no design de jogos em outros contextos (NACKE e DETERDING, 2017), neste caso, aplicado na educação. A motivação e a interação dos alunos são essenciais para a eficiência do processo de aprendizagem, principalmente quando esta se dá de forma personalizada e seguindo o ritmo particular de cada um. Manter os alunos 
motivados e promover a socialização entre eles são objetivos comuns do gamification, que tem como exemplo clássico o sistema de recompensas.

Por último, pode-se citar os MOOCs, os cursos massivos e abertos online. Existem diversas plataformas, como o edX e o Coursera, já mencionados anteriormente, que trazem inúmeros cursos online e gratuitos nas mais diversas áreas do conhecimento. A ideia é democratizar o acesso ao conhecimento e torná-lo disponível a qualquer hora, em qualquer lugar do mundo.

Considerando as metodologias e conceitos apresentados e a essência do self-paced learning, que é a aprendizagem no ritmo de cada aluno (CLARK e MAYER, 2003), é possível observar que a tecnologia é uma grande facilitadora desse processo, visto que ela fornece os meios e os recursos para a promoção desses conceitos, possibilitando aos alunos uma maior autonomia para aprender e dedicar seus esforços nas áreas de conhecimento que eles julgarem mais importantes.

\section{Metodologia da Revisão Sistemática}

Esta seção apresenta a metodologia usada na revisão sistemática, desde a estratégia de busca de artigos até a extração de dados. O objetivo principal, como mencionado anteriormente, é verificar e analisar as metodologias implementadas para a promoção e o suporte ao e-learning e ao self-paced learning no contexto do Ensino Superior. Assim, é possível conhecer o estado da arte nesse contexto específico e propor estratégias para otimizar o uso da tecnologia no processo de aprendizagem.

O procedimento adotado para a realização da revisão sistemática da literatura foi baseado na metodologia proposta por Kitchenham (2004), que segue um protocolo bem definido para a identificação, a seleção, a avaliação e a interpretação dos resultados de uma pesquisa de relevância com base em uma questão de pesquisa previamente escolhida. Este protocolo define uma série de fases que devem ser cumpridas para contemplar as etapas de pesquisa e que, considerando esta revisão sistemática em particular, podem ser descritas da seguinte forma:

- Fase 1: pesquisa na literatura por meio das bases digitais;

- Fase 2: filtragem das publicações a partir do título e do resumo;

- Fase 3: seleção de artigos relevantes para o contexto estudado;

- Fase 4: utilização dos critérios de inclusão e exclusão;

- Fase 5: seleção final das publicações das quais foram extraídos os dados.

\subsection{Questões de pesquisa}

Para esta revisão sistemática, foram consideradas as seguintes questões de pesquisa:

- Q1 - Quais são os conceitos que embasam as metodologias desenvolvidas e avaliadas para promover ou dar suporte ao e-learning e ao self-paced learning no contexto do Ensino Superior?

- Objetivo: identificar os conceitos que embasam as metodologias existentes e de que forma eles promovem ou dão suporte ao e-learning e ao self-paced learning no contexto do Ensino Superior, além de analisar a avaliação de cada uma delas.

- Q2 - Quais as dificuldades e desafios encontrados na aplicação desses conceitos e metodologias?

- Objetivo: identificar as dificuldades e desafios encontrados na aplicação desses conceitos e metodologias para traçar uma estratégia eficiente de implementação na prática.

A partir dessas questões, foi planejado e aplicado o protocolo de revisão utilizado, bem como seus testes e conduta iniciais. 


\subsection{Etapas de Pesquisa}

Para a busca de artigos e publicações científicas relacionadas ao uso da tecnologia no Ensino Superior, foram utilizadas as bases digitais online:

- Springer Link https: / / link.springer.com;

- Science Direct https: / / www.sciencedirect.com;

- IEEE Xplore Digital Library http://ieeexplore.ieee.org;

- e ACM Digital Library https://dl.acm.org/.

Essas bases foram escolhidas porque oferecem interfaces de busca bem organizadas e retornam resultados em inglês. As buscas realizadas foram feitas com base nas seguintes palavras-chave: self-paced learning; e-learning; e Higher Education. A escolha foi feita de acordo com a relevância dos resultados das buscas, com a quantidade de publicações retornadas, com o idioma da publicação, que deveria ser em inglês, e com a data de publicação, que compreende o período entre 2012 e 2018. Como mencionado na Seção 1, o período de publicação dos artigos considerados foi escolhido levando em consideração o surgimento dos MOOCs em 2012, com o lançamento do Coursera em abril, do edX em maio e o posterior lançamento da plataforma Open edX, em junho de 2013. Assim, os MOOCs representaram o surgimento de uma nova forma de aplicar o conceito de self-paced learning a partir do uso de e-learning e, portanto, foram considerados um marco importante na forma de lidar com o ensino à distância, em particular no contexto do Ensino Superior, já que grandes universidades do mundo fizeram parceria com essas plataformas, como por exemplo o MIT, a Universidade de Harvard, de Stanford e de Princeton, entre outras.

Considerando as fases de pesquisa previamente citadas e o contexto abordado neste trabalho, foram testadas várias combinações com as palavras-chave escolhidas. A string de busca que trouxe mais resultados relevantes para o tema foi:

\section{• ("Higher Education" AND "e-learning" AND "self-paced learning")}

Essa string retornou $\mathbf{8 9}$ resultados, entre artigos de revistas e conferências, que configuram a Fase 1, sendo 20 da base Science Direct, 51 da IEEE Xplore Digital Library e 18 da base Springer Link. Vale ressaltar que a ACM Digital Library não retornou nenhum resultado no contexto buscado considerando a string utilizada. A Fase 2 da revisão foi a realização da leitura do título e do resumo de cada um dos artigos retornados pela busca nas bases. A Fase 3 se caracterizou pela seleção das publicações relevantes.

\subsection{Critérios de Inclusão e Exclusão}

A partir da análise da etapa anterior, as publicações selecionadas serviram de entrada para a Fase 4, na qual, a partir dos critérios de inclusão e exclusão, foi feita a seleção daqueles que são relevantes para a lista de estudos primários. Os critérios de inclusão observados foram:

- Incluir qualquer estudo que propõe, apresenta, compara, avalia ou discute metodologias de implementação de self-paced learning e e-learning no contexto do Ensino Superior;

- Incluir qualquer estudo que propõe, apresenta, compara, avalia ou discute ferramentas de auxílio à aprendizagem no contexto do Ensino Superior por meio de self-paced learning e e-learning.

Em contrapartida, os critérios de exclusão observados foram: 
- Excluir estudos que não propõem, apresentam, comparam, avaliam ou discutem metodologias de implementação de self-paced learning e e-learning no contexto do Ensino Superior;

- Excluir estudos que não propõem, apresentam, comparam, avaliam ou discutem ferramentas de auxílio à aprendizagem no contexto do Ensino Superior por meio de self-paced learning e e-learning;

- Excluir estudos que sejam revisões sistemáticas;

- Excluir estudos que tratam da temática abordada, mas não estão inseridos no contexto do Ensino Superior.

\section{Resultados}

Após a aplicação dos critérios de seleção nos 89 artigos obtidos inicialmente, foram selecionadas 31 publicações para a Fase 5, que consiste na seleção final de publicações a partir das quais foi feita a extração de dados, sendo 9 da base Science Direct, 19 da IEEE Xplore Digital Library e 3 da base Springer Link. A Tabela 1 exibe a relação dos artigos selecionados.

Tabela 1 - Relação de artigos selecionados para a extração de dados

\begin{tabular}{|c|c|c|}
\hline Base & Fase Inicial & Fase Final \\
\hline Science Direct & 20 & 9 \\
\hline IEEE Xplore Digital Library & 51 & 19 \\
\hline Springer Link & 18 & 3 \\
\hline Total & 89 & 31 \\
\hline
\end{tabular}

Fonte: Autor

A partir dos artigos selecionados, foi feita a análise de quais são as metodologias e ferramentas de e-learning usadas para promover o conceito de self-paced learning no contexto do Ensino Superior, que países concentram sua aplicação e quais os pontos positivos oriundos de sua adoção, bem como os desafios enfrentados para implementá-las.

A princípio, os artigos foram classificados quanto ao principal conceito ou metodologia de elearning abordado. A Figura 1 sumariza os dados dessa classificação. Dos 31 trabalhos analisados:

- 11 têm o blended learning como centro de estudo (SONGKRAM et al., 2015; BAE et al., 2015; KHLAISANG e LIKHITDAMRONGKIAT, 2015; OKAZ, 2015; ALMAHASHEER, 2016; ZHIYU, 2013 HENDRADJAYA et al., 2014; CUNNINGHAM, 2016; BRALIĆ e DIVJAK, 2018; DEBNATH et al., 2014; WEI et al., 2017);

- 7 se concentram puramente no conceito de self-paced learning (HERATH et al., 2015a; HERATH et al., 2015b; GULATI, 2013; MAINA e KIHORO, 2017; CHEN et al., 2017; AMANDU et al., 2013; PEART et al., 2017); 
- 7 abordam o e-learning sem nenhum tipo de especialização (AGUDO-PEREGRINA et al., 2014; SCHULZ et al., 2014; GHAVIFEKR e MAHMOOD, 2017; KAPOOR et al., 2014; ASOODAR et al., 2016; SHAHABADI e UPLANE, 2015; GITONGA et al., 2016);

- 3 são voltados ao conceito de gamification (ALENEZI e SHAHI 2015; SCHULZ et al., 2015; LENNON et al., 2015);

- 2 abordam e-learning no contexto de dispositivos móveis, o m-learning (OYELERE et al., 2016; OOI et al., 2018);

- $\quad$ e 1 traz uma abordagem centrada no flipped learning (ZHANG et al., 2016).

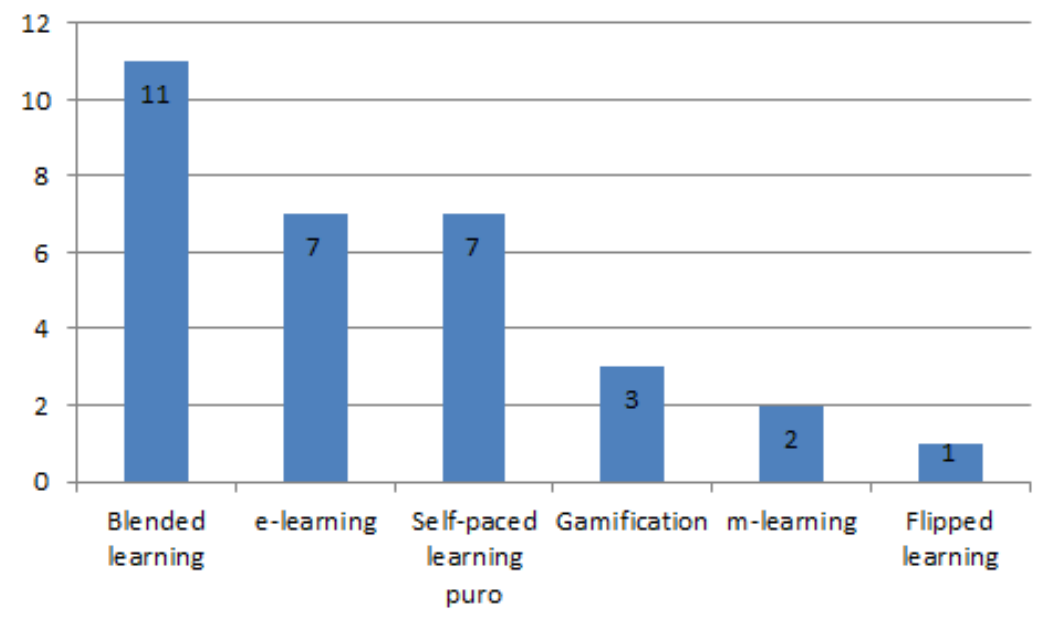

Figura 1. Classificação por Conceitos e Metodologias

Fonte: Autor

Também foi feito um levantamento dos conceitos e metodologias secundariamente citados, isto é, fora do tema central abordado. Nesse contexto, MOOCs foram citados 4 vezes (HERATH et al., 2015a; BAE et al., 2015; CUNNINGHAM, 2016; LENNON et al., 2015), hybrid learning foi mencionado em 3 artigos (KHLAISANG e LIKHITDAMRONGKIAT, 2015; KAPOOR et al., 2014; ALMAHASHEER, 2016), blended learning em 2 (LENNON et al., 2015; ZHANG et al., 2016), gamification em 1 (SCHULZ et al., 2014) e a ideia de e-learning metacognitivo apareceu em 1 artigo (GULATI, 2013).

Quanto às plataformas, nem todos os estudos foram feitos a partir da análise de uma ferramenta específica. Dos 31 artigos:

- 15 fizeram uma abordagem genérica, aplicando somente conceitos e metodologias, e não utilizaram diretamente um sistema computacional de apoio ao ensino ou aprendizagem (HERATH et al., 2015a; GULATI, 2013; BAE et al., 2015; MAINA e KIHORO, 2017; SCHULZ et al., 2014, OYELERE et al., 2016, OKAZ, 2015; ZHIYU, 2013; HENDRADJAYA et al., 2014; SHAHABADI e UPLANE, 2015; SCHULZ et al., 2015; LENNON et al., 2015; OOI et al., 2018; DEBNATH et al., 2014; PEART et al., 2017);

- 6 se basearam no Moodle (HERATH et al., 2015b; AGUDO-PEREGRINA et al., 2014; ASOODAR et al., 2016; AMANDU et al., 2013; CUNNINGHAM, 2016; BRALIĆ e DIVJAK, 2018); 
- 3 fizeram análises a partir de ferramentas desenvolvidas pelos autores ou pela universidade que foi objeto de estudo (SONGKRAM et al., 2015; KHLAISANG e LIKHITDAMRONGKIAT, 2015; KAPOOR et al., 2014);

- 2 trabalharam diretamente com o Blackboard (ALMAHASHEER, 2016; ZHANG et al., 2016);

- e alguns sistemas apareceram apenas 1 vez, como SPeCTRUM (GHAVIFEKR e MAHMOOD, 2017), FishBuddy (Chen et al., 2017), Edmodo (GITONGA et al., 2016) e Star C (WEI et al., 2017).

Além das plataformas desenvolvidas com o propósito de apoiar a aprendizagem ou gerenciar o ensino, o jogo Second Life também apareceu uma vez como principal ferramenta de estudo (ALENEZI e SHAHI, 2015). A Figura 2 sumariza os dados dessa classificação.

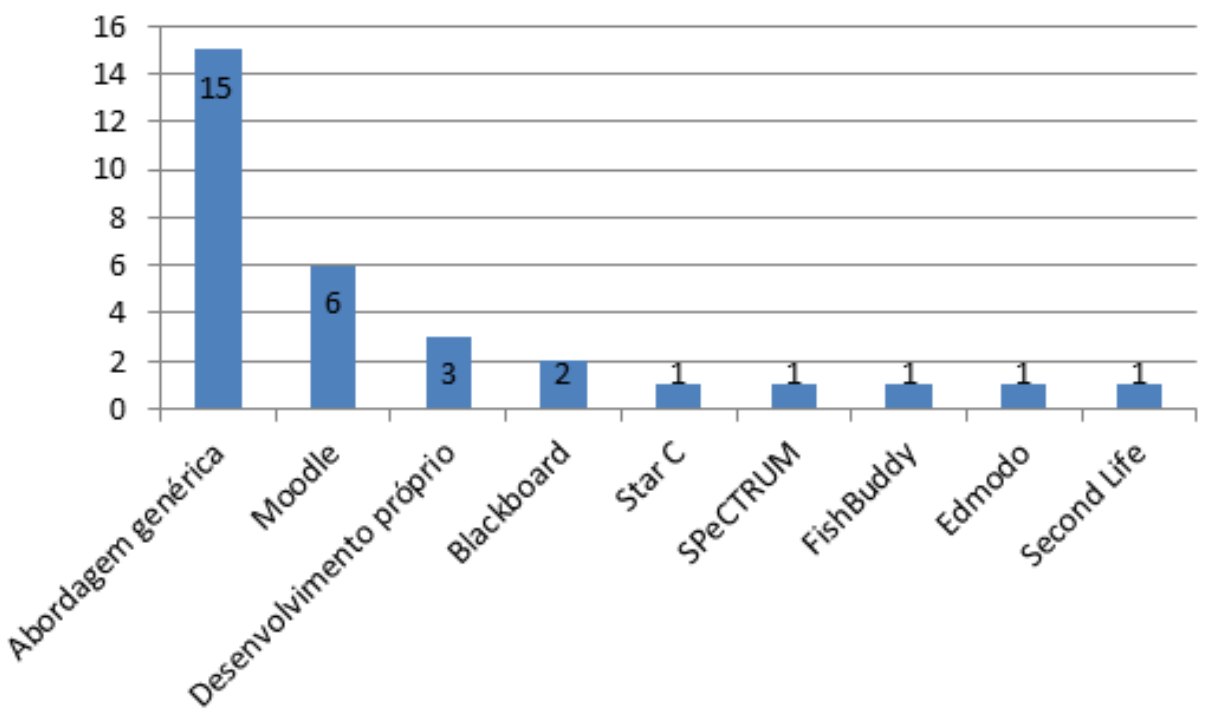

Figura 2. Classificação por plataformas utilizadas Fonte: Autor

Sobre os pontos positivos da utilização do conceito de e-learning como meio de promoção do self-paced learning, os mais citados ou mencionados foram: a flexibilidade no processo de aprendizagem, com 8 citações; a aprendizagem colaborativa e a acessibilidade, com 7 menções cada; a motivação, os métodos de avaliação e o material estruturado, que foram citados 4 vezes cada; e o feedback contínuo, o maior envolvimento e a melhoria das notas dos alunos, mencionados 3 vezes cada. A Figura 3 traz um gráfico com o resumo dos pontos positivos mais citados. 


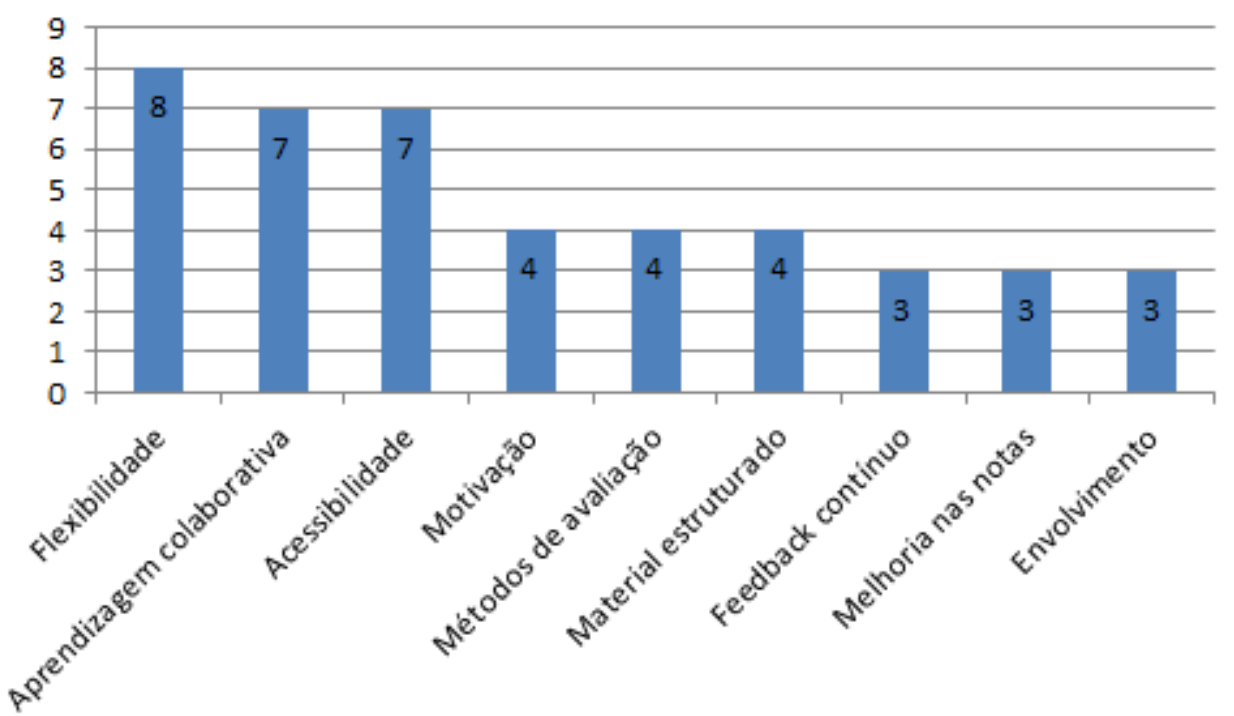

Figura 3. Pontos positivos mais citados

Fonte: Autor

Por outro lado, os principais desafios citados foram: a falta de interação entre os alunos e entre professores e alunos, com 5 citações; a necessidade de treinamento para utilização das plataformas, com 4 menções; a limitação no conhecimento tecnológico, citada 3 vezes; e a infraestrutura limitada, a ausência do professor no sistema de feedback e a falta de coerência dos materiais utilizados, mencionadas 2 vezes cada. A Figura 4 apresenta graficamente os principais desafios encontrados.

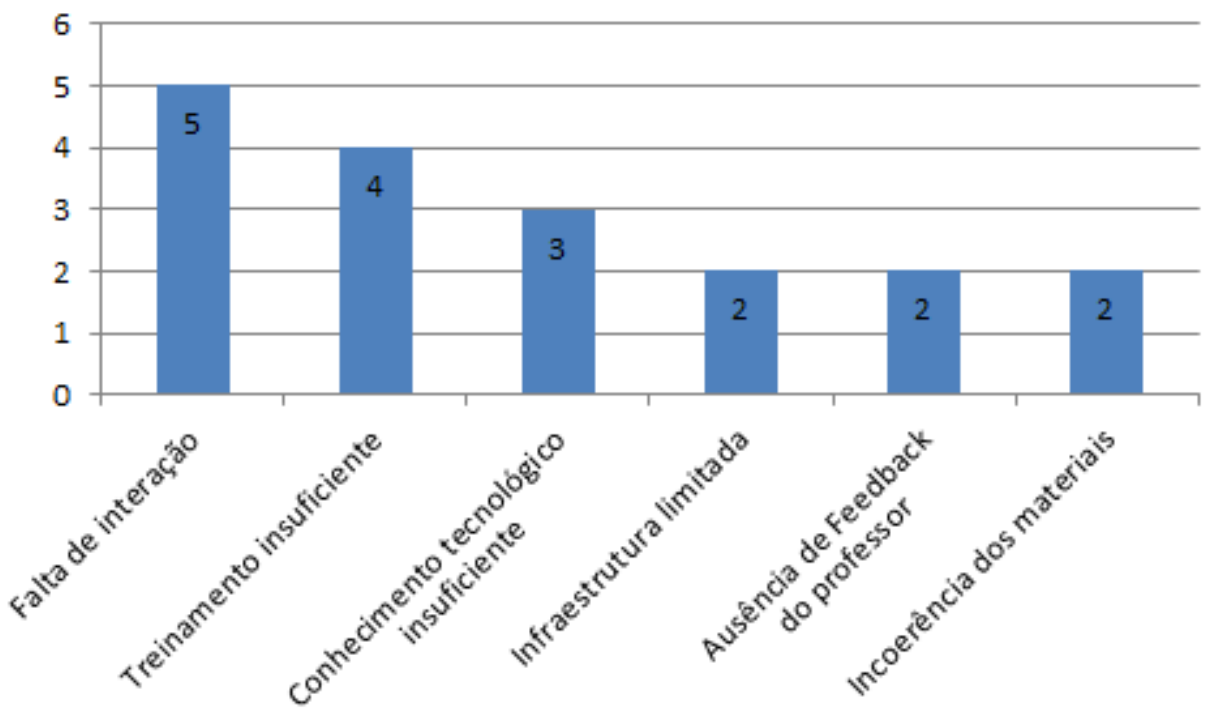

Figura 4. Principais desafios encontrados

Fonte: Autor

Por fim, os artigos apresentaram grande diversidade quanto à nacionalidade. Os 31 artigos analisados tiveram origem em 20 países diferentes:

- 3 são da China (CHEN et al. 2017, ZHIYU 2013, WEI et al. 2017);

- 3 da Índia (GULATI, 2013; KAPOOR et al., 2014; SHAHABADI e UPLANE, 2015); 
- 2 da Arábia Saudita (ALENEZI e SHAHI, 2015; ALMAHASHEER, 2016);

- 2 da Austrália (BAE et al., 2015; LENNON et al., 2015);

- 2 da Malásia (GHAVIFEKR e MAHMOOD, 2017; OOI et al., 2018);

- 2 da Noruega (SCHULZ et al., 2014; SCHULZ et al., 2015);

- 2 do Quênia (MAINA e KIHORO, 2017; GITONGA et al. 2016);

- 2 do Sri Lanka (HERATH et al., 2015a; HERATH et al., 2015b);

- 2 da Tailândia (SONGKRAM et al., 2015; KHLAISANG e LIKHITDAMRONGKIAT, 2015);

- 1 de Bangladesh (DEBNATH et al., 2014);

- 1 da Croácia (BRALIĆ e DIVJAK, 2018);

- 1 do Egito (OKAZ, 2015);

- 1 da Espanha (AGUDO-PEREGRINA et al., 2014);

- 1 dos Estados Unidos (ZHANG et al., 2016);

- 1 da Finlândia (OYELERE et al., 2016);

- 1 da Indonésia (HENDRADJAYA et al., 2014);

- 1 do Irã (ASOODAR et al., 2016);

- 1 de Omã (AMANDU et al., 2013);

- 1 do Reino Unido (PEART et al., 2017);

- 1 da Suécia (CUNNINGHAM, 2016).

\section{Discussão}

Esta seção apresenta a discussão dos resultados observados a partir das questões de pesquisa elaboradas.

\subsection{Q1 - Quais são os conceitos que embasam as metodologias desenvolvidas e avaliadas para promover ou dar suporte ao e-learning e ao self-paced learning no contexto do Ensino Superior?}

Como apresentado na Seção 4, a metodologia de blended learning foi o centro de estudo de 11 dos 31 artigos analisados. Isso ocorre principalmente porque sua ideia principal é inserir elementos de e-learning no ensino tradicional (OSORIO GOMÉZ e DUART, 2012) para personalizar e estender o processo de aprendizagem para fora da sala de aula, o que vai de encontro às necessidades das universidades. O trabalho apresentado por Debnath et al. (2014), por exemplo, avalia a implantação de blended learning em um curso de engenharia em Bangladesh para que os alunos possam ter acesso mais simples e facilitado aos materiais utilizados no ensino, aumentando sua autonomia e independência para que eles sejam capazes de fazer uma autoavaliação sobre o nível de compreensão da matéria. 
O artigo de Bralić e Divjak (2018) propõe a utilização de blended learning a partir da integração de um MOOC a uma sala de aula tradicional como um modelo a ser adotado no contexto do Ensino Superior. Segundo estudos qualitativos realizados pelos autores, os alunos apreciaram essa abordagem principalmente pelo processo de aprendizagem embasado no conceito de self-paced learning, mas ressaltaram a autodisciplina necessária para que ele funcione. Outro fator interessante observado foi a adesão dos alunos que não estudam em tempo integral, uma vez que, com a flexibilidade de horário oferecida pelo modelo, eles conseguiram conciliar os estudos com o trabalho. Além disso, o artigo trouxe ainda três conceitos de níveis de aprendizagem:

- Deep, que é caracterizado por alunos que buscam compreender as questões e interagir de forma crítica com os conteúdos dos materiais de ensino, relacionando ideias a experiências e conhecimentos prévios e examinando a lógica dos argumentos por meio da relação das evidências apresentadas com suas conclusões;

- Strategic, que é uma abordagem voltada para a obtenção de notas altas nas avaliações e tem ênfase nas habilidades dos alunos;

- E surface, que é caracterizada apenas pela memorização e pelo isolamento de ideias diferentes.

Segundo o levantamento dos autores, o uso de MOOCs motiva os alunos a aprenderem segundo uma abordagem profunda (deep), pois eles relacionaram as descobertas do curso online com o curso regular e foram instigados a pensar de forma crítica sobre o conteúdo visto.

Contudo, muitos alunos se sentiram motivados apenas pelas notas e por motivos extrínsecos, que são características das abordagens estratégica (strategic) e superficial (surface). Por fim, o idioma dos MOOCs foi apontado como um desafio e, ao mesmo tempo, como um fator de incentivo, uma vez que a maioria dos cursos está disponível em inglês e o estudo foi realizado na Croácia.

No trabalho de Gulati (2013), é sugerido um sistema especialista de e-learning colaborativo com base em um software que funcione como agente cognitivo, isto é, o sistema é capaz de sugerir ao aluno conteúdos encontrados na Internet de acordo com seu perfil e aprender a partir de suas escolhas. A ideia principal é que o processo de aprendizagem aconteça também de maneira informal, se estendendo para além do ambiente acadêmico a partir do interesse dos alunos, uma vez que o sistema deve analisar as aptidões dos usuários para indicar fontes e conteúdos que as atendam. A essa abordagem, segundo o autor, é dado o nome de e-learning metacognitivo e o sistema nela embasado deve ser autônomo, responsivo, baseado no aprendizado de máquina, adaptativo, colaborativo, cooperativo e contínuo. Apesar da sugestão do sistema e das características que ele deve contemplar, não foi desenvolvido no trabalho em questão um protótipo ou uma plataforma real que o implementasse.

Considerando o fator motivacional, que é um dos principais objetivos do uso de gamification no processo de aprendizagem, o trabalho de Lennon (2015) relata a implementação do conceito em um estudo sobre células de energia solar. A ideia é manter os alunos motivados e aumentar o engajamento com o curso, com o conteúdo e com os demais alunos. Os autores sugerem ainda a incorporação do conceito de gamification aos MOOCs como solução para um problema de evasão apontado no estudo, que indica que, de forma geral, a taxa de conclusão de cursos nesse formato é de apenas $7 \%$, o que é atribuído diretamente à falta de engajamento e motivação dos alunos. No caso específico sobre o estudo de células de energia solar, foi criado um ranking de eficiência para que os alunos competissem entre si para ver quem seria capaz de criar células mais eficientes. Essa medida se mostrou positiva quanto ao engajamento, mas também mostrou um lado negativo da competitividade trazida pelo método: os alunos que não conseguiram uma boa colocação no ranking afirmaram, por meio de uma pesquisa conduzida informalmente pelos autores, que não gostaram do sistema competitivo. Assim, a incorporação de elementos de jogos no contexto da educação se mostra capaz de 
motivar os alunos, mas deve ser feita de forma planejada e cuidadosa para que não gere um efeito contrário.

O trabalho de Zhang et al. (2016) propõe a aplicação de flipped learning e blended learning em larga escala em um curso de sistemas de informação da Northern Arizona University, nos Estados Unidos. O curso utiliza o Blackboard como ferramenta de e-learning e o método de Cornell Notes como base para aplicar um modelo de flipped learning que consiste no fichamento e no resumo, escritos à mão, do material digital lido para evitar a distribuição de cópias do trabalho individual do aluno, além de atividades em sala de aula feitas a partir do que foi previamente estudado, como exercícios e debates. Contudo, a utilização do método foi um ponto controverso no estudo levantado pelo trabalho, pois enquanto alguns alunos o enxergaram como uma forma de revisar e auxiliar na assimilação do conteúdo, outros consideraram a necessidade de escrever em um papel as informações já disponíveis digitalmente como perda de tempo e trabalho. Por fim, os autores ressaltam que, de acordo com os resultados obtidos por meio de questionários aplicados aos participantes do estudo, as características dos instrutores não foram consideradas importantes, o que reforça a ideia de que nessas metodologias o aluno é o protagonista do processo de aprendizagem.

\subsection{Q2 - Quais as dificuldades e desafios encontrados na aplicação desses conceitos e metodologias?}

De acordo com o apresentado na Seção 4, a flexibilidade foi o ponto positivo das metodologias que aplicam e-learning mais citado dentre os 31 artigos, com 8 menções. De fato, esta é uma característica marcante desse conceito, pois o conteúdo fica disponível online para que o aluno o acesse onde e quando quiser, o que está diretamente relacionado à acessibilidade, outro ponto positivo citado 7 vezes na revisão. O trabalho de Cunningham (2016) apresenta a importância desse fator em um estudo sobre as lacunas de conhecimento presentes nas universidades do Quênia. A partir de cursos ministrados com o apoio das plataformas Moodle e Blackboard, foi feita uma análise qualitativa sobre a eficiência da metodologia implementada que evidenciou as limitações de infraestrutura encontradas no país, o que dificulta a utilização de ferramentas de e-learning que são importantes para promover o self-paced learning. A pesquisa mostra que simplesmente inserir a tecnologia no meio de ensino não resulta na melhoria dos padrões de ensino e aprendizagem ou em uma maior absorção de conhecimento. Tanto os benefícios quanto os desafios independem das tecnologias usadas, portanto é necessário pesquisar abordagens psicológicas que colocam o aluno como centro do processo e utilizar conceitos e metodologias apropriados para cada situação em particular.

A falta de interação foi o desafio mais citado dentre os trabalhos pesquisados, como apresentado na Figura 4. Contudo, ela vai de encontro a dois dos pontos positivos mais mencionados na revisão, que são a aprendizagem colaborativa e o envolvimento dos alunos. O trabalho de Ghavifekr e Mahmood (2017) apresenta esses três pontos concomitantemente, enfatizando que a falta de interação social e o isolamento dos alunos não impede que o processo de aprendizagem se dê de forma colaborativa e que os alunos se envolvam com ele, mas também alerta que esses fatores não significam necessariamente interação entre eles. Como forma de vencer esse desafio, o autor sugere a utilização de elementos presentes no elearning síncrono, como o uso de chats online, por exemplo, para aproximar os alunos e incentivar a troca de informações em tempo real.

O treinamento para utilização da ferramenta e o conhecimento tecnológico necessário para usar métodos de e-learning são desafios que estão diretamente relacionados entre si. Novamente citando o trabalho de Cunningham (2016), que foi realizado no Quênia, percebe-se o quanto esses desafios estão diretamente relacionados aos professores e às instituições, e não necessariamente aos alunos. A maior resistência encontrada quanto à utilização de métodos de e-learning no que tange a esses desafios foi de professores que não se sentem confortáveis com o uso da tecnologia e de instituições de ensino que enxergam o tempo e os recursos 
gastos com o treinamento para utilização das ferramentas como obstáculos, o que é corroborado pelos estudos de Herath et al. (2015b), por exemplo, que traz o contexto da Universidade de Wayamba, no Sri Lanka, como estudo de caso.

\section{Considerações Finais e Trabalhos Futuros}

Este trabalho abordou a utilização de métodos e ferramentas de e-learning para promover o conceito de self-paced learning no contexto do Ensino Superior por meio de uma revisão sistemática da literatura. A partir dos resultados apresentados na Seção 4 e das discussões feitas na Seção 5, é possível observar que há diversos elementos de e-learning implementados em universidades por todo o mundo, além de vários modelos propostos pelos autores dos artigos visitados que ainda precisam ser desenvolvidos.

A diversidade geográfica observada mostra que a inserção da tecnologia na educação não é uma exclusividade dos países desenvolvidos, mas sim uma necessidade principalmente para os países em desenvolvimento que, a partir da utilização de métodos e ferramentas de e-learning, conseguem levar aos alunos os conteúdos e materiais de qualidade mais recentes e de maior relevância educacional segundo as melhores universidades do mundo, principalmente por meio dos MOOCs. Contudo, é preciso levar em consideração as limitações de infraestrutura desses países, que constituem um desafio para a implementação efetiva das plataformas e ferramentas propostas. Vale observar, ainda, a ausência de trabalhos brasileiros dentre os estudados.

Existem vários pontos positivos na utilização das metodologias e conceitos apresentados, como mostrado na Figura 3, que devem ser observados e considerados na hora de desenvolver um método para ser aplicado em uma ferramenta de promoção da aprendizagem segundo o conceito de self-paced learning. Dentre as metodologias apresentadas, o blended learning se mostrou o mais utilizado, já que mantém os elementos do ensino tradicional que também são positivos no processo de aprendizagem. Para manter os alunos motivados e engajados, a utilização de gamification se mostrou a alternativa mais buscada.

Para o desenvolvimento de uma ferramenta, é preciso levar em consideração os desafios levantados nesta revisão sistemática da literatura, com destaque para a falta de interação observada nos modelos existentes, que foi o fator mais citado entre os artigos estudados. Para vencer essa barreira, é preciso pensar em elementos de e-learning síncrono que podem ser aplicados no desenvolvimento e aproveitar o tempo presencial contemplado pelas metodologias de blended learning, tornando o processo de aprendizagem mais completo e agradável para o aluno, que é o seu protagonista.

Desse modo, a partir dos desafios identificados nesta revisão sistemática da literatura e como visão de próximos passos, propõe-se o desenvolvimento de uma ferramenta de apoio à aprendizagem no contexto do Ensino Superior que promova o conceito de self-paced learning, considerando os principais desafios e pontos positivos e negativos levantados. Esta ferramenta deve ser capaz de apoiar a tomada de decisão do aluno no que diz respeito aos conteúdos a serem abordados e indicar possíveis caminhos a serem trilhados de acordo com suas características, objetivos e conhecimentos prévios. Uma proposta semelhante foi feita no modelo trazido por Gulati (2013) que, apesar de não ter sido implementado, aproxima-se bastante da ideia de desenvolvimento proposta a partir deste trabalho.

Para o desenvolvimento da ferramenta, será utilizada como ponto de partida a plataforma Open edX (https://github.com/edx/edx-platform), que é uma iniciativa de software livre utilizada como base em todos os cursos do edX. Esta escolha se deve, principalmente, ao fato da plataforma ser aberta, possuir um ecossistema de apoiadores amplo e ativo e ser bem documentada. Dentre as novas funcionalidades a serem desenvolvidas e aquelas que já existem, mas que podem ser aperfeiçoadas, destacam-se:

- Personalização do ambiente de aprendizagem: o usuário da ferramenta, seja professor, tutor ou aluno, poderá personalizá-la para melhor atender as suas 
necessidades, tanto na forma de utilização quanto no conteúdo. Será possível, por exemplo, filtrar conteúdos de acordo com a área de conhecimento, criar tópicos de acordo com o interesse, interagir com usuários que tenham interesses semelhantes e integrar a ferramenta às contas de redes sociais;

- Criação de turmas: um professor ou tutor poderá criar uma turma, com a qual vai poder compartilhar e disponibilizar atividades e conteúdos de diversos formatos (vídeos, áudios, textos e planilhas, por exemplo, em arquivos editáveis ou somente de leitura) e por meio de diversas plataformas/redes sociais, com plug-ins e links;

- Aperfeiçoamento do sistema de avaliação entre alunos: a ferramenta vai dispor de um sistema que possibilita a avaliação das atividades não só por um tutor ou professor, mas também entre os alunos, sendo esta feita de forma anônima (caso desejado), tanto para quem avalia quanto para quem é avaliado;

- Painel de controle: cada usuário, seja professor ou aluno, terá em sua página um painel de controle que traz dados estatísticos e informações detalhadas, como desempenho, progresso, áreas de domínio e sugestões de conteúdo, por exemplo. Essas informações devem ser apresentadas de forma clara e de fácil visualização, por meio de tabelas e gráficos, e serão utilizadas para explorar elementos de gamification como, por exemplo, a atribuição de medalhas para os alunos de acordo com o progresso obtido nas áreas de conhecimento;

- Fóruns de discussão: será possível participar de fóruns de discussão que englobam os mais diversos níveis de usuário, desde fóruns fechados para a turma ou sobre um conteúdo específico, até discussões que potencialmente envolvam todos os usuários. Também deve ser feita uma avaliação sobre a participação dos usuários nesses espaços;

- Publicação de trabalhos e atividades: a ferramenta disponibilizará uma opção para tornar os trabalhos e projetos públicos para a comunidade, viabilizando uma maior interação e incentivando o compartilhamento do conhecimento;

- Auxílio na tomada de decisão: esta funcionalidade não foi observada nesta revisão em nenhum estudo que contemple o contexto do Ensino Superior. Com ela, esperase que a ferramenta seja capaz de apoiar a tomada de decisão do aluno quanto ao conteúdo a ser abordado e de indicar possíveis caminhos a serem trilhados de acordo com seu perfil, suas características e seus conhecimentos prévios, oferecendo assim mais do que uma trajetória de estudo, isto é, permitindo abordagens não lineares.

Portanto, em linhas gerais, a visão de trabalho futuro consiste em estender o suporte ferramental fornecido por plataformas de e-learning, criando novas funcionalidades e aprimorando as já existentes para apoiar, de forma mais ativa e inteligente, as atividades de self-paced learning. Desenvolver ferramentas capazes de oferecer trilhas diferenciadas de aprendizagem, flexíveis e não lineares, adequadas à realidade do estudante, pode ser fundamental para atingir esse objetivo.

\section{Referências}

AGUDO-PEREGRINA, Ángel F.; HERNÁNDEZ-GARCÍA, Ángel; PASCUAL-MIGUEL, Félix J. Behavioral intention, use behavior and the acceptance of electronic learning systems: Differences between higher education and lifelong learning. Computers in Human Behavior, v. 34, p. 301-314, 2014.

ALENEZI, Ahmed Maajoon; SHAHI, Krishna Kirti. Interactive e-learning through second life with blackboard technology. Procedia-Social and behavioral sciences, v. 176, p. 891-897, 2015. 
ALMAHASHEER, Muneerah Badr. A study of faculty motivation to develop and deliver a basic blended ecourse in community college at the University of Dammam, Saudi Arabia. In: SAI Computing Conference (SAI), 2016. IEEE, p. 788-795, 2016.

AMANDU, Gerald Matua; MULIIRA, Joshua Kanaabi; FRONDA, Dennis Cayaban. Using moodle e-learning platform to foster student self-directed learning: Experiences with utilization of the software in undergraduate nursing courses in a Middle Eastern university. Procedia-Social and Behavioral Sciences, v. 93, p. 677-683, 2013.

ASOODAR, Maryam; VAEZI, Shahin; IZANLOO, Balal. Framework to improve e-learner satisfaction and further strengthen e-learning implementation. Computers in Human Behavior, v. 63, p. 704-716, 2016.

$\mathrm{BAE}$, E. et al. Framework to improve delivery methods in higher education through online learning. In: Engineering Education (ICEED), 2015 IEEE 7th International Conference on. IEEE, p. 130-134, 2015.

BERGMANN, Jonathan; SAMS, Aaron. How the flipped classroom is radically transforming learning. The Daily Riff, v. 2012, p. 1-3, 2012.

BRALIĆ, Antonia; DIVJAK, Blaženka. Integrating MOOCs in traditionally taught courses: achieving learning outcomes with blended learning. International Journal of Educational Technology in Higher Education, v. 15, n. 1, p. 2, 2018.

CHANDLER, D. L. (2014). Study: Online classes really do work. http://news.mit. edu/2014/study-shows-online-courses-effective-0924. Último acesso em: 11/10/2017.

CHEN, Jingjing et al. FishBuddy: Promoting Student Engagement in Self-Paced Learning through Wearable Sensing. In: Smart Computing (SMARTCOMP), 2017 IEEE International Conference on. IEEE, 2017. p. 1-9.

CLARK, Ruth Colvin; MAYER, Richard E. E-learning and the science of instruction: Proven guidelines for consumers and designers of multimedia learning. Performance Improvement, v. 42, n. 5, p. 41-43, 2003.

Coursera Inc. Coursera, about us. Disponível em: https://about.coursera.org/. Último acesso em: 10/05/2018.

CUNNINGHAM, Miriam. Technology-Enhanced Learning in Kenya Universities: Influences on Wider Adoption and Take Up. IEEE Technology and Society Magazine, v. 35, n. 3, p. 28-35, 2016.

DEBNATH, B. C. et al. Learning and evaluation of blended learning approach for ICT undergraduate students. In: Electrical Engineering and Information \& Communication Technology (ICEEICT), 2014 International Conference on. IEEE, p. 1-6, 2014.

edX Inc. edX, about us. Disponível em: https://www.edx.org/about-us. Último acesso em: 10/05/2018.

GHAVIFEKR, Simin; MAHMOOD, Hazline. Factors affecting use of e-learning platform (SPeCTRUM) among University students in Malaysia. Education and Information Technologies, v. 22, n. 1, p. 75-100, 2017.

GITONGA, Rhoda; MUURO, Maina; ONYANGO, George. Technology integration in the classroom: A case of students experiences in using Edmodo to support learning in a blended classroom in a Kenyan University. In: IST-Africa Week Conference, 2016. IEEE, p. 1-8, 2016.

GULATI, Neha. Framework for cognitive agent based expert system for metacognitive and collaborative ELearning. In: Innovation and Technology in Education (MITE), 2013 IEEE International Conference in MOOC. IEEE, p. 421-426, 2013.

HENDRADJAYA, Bayu et al. The application model of learning management system quality in asynchronous blended learning system. In: Electrical Engineering and Computer Science (ICEECS), 2014 International Conference on. IEEE, p. 223-228, 2014.

HERATH, Chaminda P.; THELIJJAGODA, Samantha; GUNARATHNE, W. K. T. M. Stakeholders' psychological factors affecting E-learning readiness in higher education community in Sri Lanka. In: Ubi-Media Computing (UMEDIA), 2015 8th International Conference on. IEEE, p. 168-173, 2015a.

HERATH, Chaminda P.; WEERAKKODY, W. J. S. K.; GUNARATHNE, W. K. T. M. Infrastructure factors affection for E-learning practice of students in Wayamba University of Sri Lanka: Case study: Makandura premises. In: Ubi-Media Computing (UMEDIA), 2015 8th International Conference on. IEEE, p. 196-201, 2015b. 
HRASTINSKI, Stefan. Asynchronous and synchronous e-learning. Educause quarterly, v. 31, n. 4, p. 51-55, 2008.

KAPOOR, Amita; KABRA, Sneha; DUA, Himani. Development, use and impact of e-learning based modules for teaching electronics: To undergraduate girl students: A case study. In: MOOC, Innovation and Technology in Education (MITE), 2014 IEEE International Conference on. IEEE, 2014. p. 215-218.

KHLAISANG, Jintavee; LIKHITDAMRONGKIAT, Maneerat. E-learning system in blended learning environment to enhance cognitive skills for learners in higher education. Procedia-Social and Behavioral Sciences, v. 174, p. 759-767, 2015.

KITCHENHAM, Barbara. Procedures for performing systematic reviews. Keele, UK, Keele University, v. 33, n. 2004, p. 1-26, 2004.

LENNON, Alison; ABBOTT, Malcolm; MCINTOSH, Keith. Chasing higher solar cell efficiencies: Engaging students in learning how solar cells are manufactured. In: Teaching, Assessment, and Learning for Engineering (TALE), 2015 IEEE International Conference on. IEEE, p. 267-271, 2015.

MAINA, Elizaphan Muuro; KIHORO, John M. Learner experience of e-learning mode in institutions of higher learning: A case of Kenyan Universities. In: IST-Africa Week Conference (IST-Africa), 2017. IEEE, p. 1-9, 2017.

MITRA, Sugata. The future of learning. In: Proceedings of the Third (2016) ACM Conference on Learning@ Scale. ACM, p. 61-62, 2016.

MOTA, Ronaldo. Inovação e aprendizagem independente na educação básica. Ciência e Natura, v. 36, 2014.

MOTA, Ronaldo; SCOTT, David. Educando para inovação e aprendizagem independente. Elsevier Editora, 2014.

NACKE, Lennart E.; DETERDING, Christoph Sebastian. The maturing of gamification research. Computers in Human Behaviour, p. 450-454, 2017.

OKAZ, Abeer Ali. Integrating blended learning in higher education. Procedia-Social and Behavioral Sciences, v. 186, p. $600-603,2015$.

OOI, Keng-Boon; HEW, Jun-Jie; LEE, Voon-Hsien. Could the mobile and social perspectives of mobile social learning platforms motivate learners to learn continuously?. Computers \& Education, v. 120, p. 127-145, 2018.

OSORIO GÓMEZ, Luz Adriana; DUART, Josep M. A hybrid approach to university subject learning activities. British Journal of Educational Technology, v. 43, n. 2, p. 259-271, 2012.

OYELERE, Solomon S. et al. Discovering students mobile learning experiences in higher education in Nigeria. In: Frontiers in Education Conference (FIE), 2016 IEEE. IEEE, p. 1-7, 2016.

PEART, Daniel J. et al. Student use and perception of technology enhanced learning in a mass lecture knowledge-rich domain first year undergraduate module. International Journal of Educational Technology in Higher Education, v. 14, n. 1, p. 40, 2017.

SCHULZ, Renée; ISABWE, Ghislain Maurice; REICHERT, Frank. Supporting teachers' needs within and through E-learning systems. In: Web and Open Access to Learning (ICWOAL), 2014 International Conference on. IEEE, p. 1-4, 2014.

SCHULZ, Renee; ISABWE, Ghislain Maurice; REICHERT, Frank. Investigating teachers motivation to use ICT tools in higher education. In: Internet Technologies and Applications (ITA), 2015. IEEE, p. 62-67, 2015.

SHAHABADI, Mehdi Mehri; UPLANE, Megha. Synchronous and asynchronous e-learning styles and academic performance of e-learners. Procedia-Social and Behavioral Sciences, v. 176, p. 129-138, 2015.

SONGKRAM, Noawanit et al. E-learning system to enhance cognitive skills for learners in higher education. Procedia-Social and Behavioral Sciences, v. 174, p. 667-673, 2015.

WEI, Yitong et al. Blended Learning versus Traditional Learning: A Study on Students' Learning Achievements and Academic Press. In: Educational Technology (ISET), 2017 International Symposium on. IEEE, p. 219-223, 2017. 
ZHANG, Dongsong et al. Can e-learning replace classroom learning?. Communications of the ACM, v. 47, $\mathrm{n}$. 5, p. $75-79,2004$.

ZHANG, Yulei; DANG, Yan; AMER, Beverly. A Large-Scale Blended and Flipped Class: Class Design and Investigation of Factors Influencing Students' Intention to Learn. IEEE Trans. Education, v. 59, n. 4, p. 263-273, 2016.

ZHIYU, Li. Exploring electrical engineering curriculum teaching reformation based on blended learning mode. In: Computer Science \& Education (ICCSE), 2013 8th International Conference on. IEEE, p. 1058-1062, 2013.

Recebido em agoto de 2018

Aprovado para publicação em dezembro de 2018

\section{José Luiz Machado Morais}

Programa de Mestrado Profissional em Inovação Tecnológica - Universidade Federal de São Paulo UNIFESP, Brasil, j.morais@unifesp.br

\section{Arlindo Flavio da Conceição}

Programa de Mestrado Profissional em Inovação Tecnológica - Universidade Federal de São Paulo UNIFESP, Brasil, arlindo.conceicao@unifesp.br 